\title{
Gender Differences in Terms of Recalling Treatment Instructions - An in Vivo Study
}

Authors

\author{
Rashmi Mittal ${ }^{1}$, Aditya Kapoor ${ }^{1}$, Khurshid A. Mattoo ${ }^{2}$ \\ ${ }^{1}$ Post graduate, Department of Prosthodontics, Swami Vivekananda Subharti University, Meerut, India \\ ${ }^{2}$ Assistant Professor, Department of Prosthetic Dental Sciences, College of dental sciences, Jazan
} University, (KSA)

Corresponding Author

Dr Khurshid A Mattoo

Email:drkamattoo@rediffmail.com

\begin{abstract}
Medical and dental treatments require effective communication, both quantitatively and qualitatively to accomplish success. Instructions given to the patient are essential components of ensuring patient compliance. For some, treatment procedures, instructions are numerous and difficult. Nature has distributed abilities between sexes among humans. This study aims to find the gender differences about their ability to retain the instructions given to them during prosthodontic treatment while also determining the efficacy of such instructions.

Materials and Methods: Ninety completely edentulous patients were rehabilitated with complete denture prosthesis. A list of instructions pertaining to education and motivation of such patients were tabulated and divided into five categories (general, treatment needs, specific, significant and miscellaneous). At two different follow-up visits, the patients were asked to recall the instructions in various categories. The efficacy of the followed instructions was determined by recording of denture plaque index. Paired ' $t$ ' test was applied for comparison between the 1 st and the 2 nd appointment recall of instructions. Significant difference was determined at $P<0.05$ level between various categories.

Results: Between various categories, general instructions were recalled more than other categories but at the second recall. Between genders, female patients recalled more instructions than males at the second appointment.

Conclusion: This study concludes that the ability of male and female gender differs and the factors associated with it should be taken into consideration before depending on patients to follow treatment instructions on their own.

Keywords: patient compliance, complete denture, treatment needs, patient instructions.
\end{abstract}

\section{Introduction}

Patient satisfaction with a particular medical or dental treatment is dependent on multiple factors out of which most are associated with practitioner while some are largely dependent on the patient himself. Most treatments at some or all stages depend largely on patient's ability to understand and follow the instructions given to them. These instructions may be given either prior, during or after treatment or even a combination. Most medical specialties like physicians, surgeons, ophthalmologists, orthopedicians, pediatricians and even gynecologists have to rely on patient's ability to 
understand and follow the instructions comprehensively. Complex and serious problems require hospitalization where it is easy to monitor patient compliance. The same is not the case when patients are not under the observation of the clinician, although they may claim they have understood the instructions and will follow them.

Replacing natural teeth is a daunting task. Natural teeth when lost renders a person handicapped from multiple functions like mastication, phonetics, aesthetic, pleasure of taste and flavors and more importantly social and psychological related factors. Any prosthodontist, who wants to fulfill these vast areas of patient's expectation and satisfaction must possess a high level of communicative skills. Though patient's satisfaction has multicausal character, like personality, attitude toward treatment, prior experience and education and motivation for wearing and maintaining a denture, ${ }^{1}$ patient education in the field of prosthodontic has been reported to be conspicuous by its absence. ${ }^{2}$ Denture patients use their prosthesis more than prescribed which opposes accomplishing preservation of remaining oral tissues. ${ }^{3,4}$ Patients attitude towards the instructions given by their specialist are largely based about his perception of the health problem he has. Every medical or dental practitioner while giving instructions to his patients has two goals in mind: short term and long term. From the patients view, priorities within a treatment differ between sexes, race, population or community. This clinical study is primarily aimed to determine the comparative gender differences at two different recall appointments about the treatment related instructions given to them during routine Prosthodontic treatment. While doing so, the study would also determine the existing status and practice of patient education and motivation by internees in undergraduate dental school and would be able to also identify instructions which are easy to remember and difficult to follow.

\section{Materials and Methods}

Before piloting the clinical study, an approval was sought from the ethical committee of the university and all clinical procedures, including those for prosthesis fabrication were conducted in accordance with the ethical standards of the ethical Committee on Human
Experimentation of the institution which follows ethical standards of "Helsinki Declaration of 1975". 5 A preliminary ancillary survey conducted on three hundred and twenty undergraduate students from different dental schools of North India pursuing internship (had completed their required quota of clinical cases in complete denture prosthesis) revealed that average number of instructions given by them to their patients was significantly very low. Data analysis of this survey revealed that the reasons for such low quantity of the instruction deliverance of the patients was due to their high number, degree of difficulty in understanding, interpreting and memorizing. This preliminary survey incited to carry the present study in which ninety completely edentulous patients seeking complete denture prosthesis on their will, were selected from the general OPD of the post graduate wing of the Department of Prosthodontics.

Ninety consentful patients (45 males and 45 females) in the age group of 50 to 60 years, whose attitude was philosophical, were first time denture wearers and were able to read/understand in either English or local language, were not suffering from any systemic medical problem, had no history of dementia or associated disorders, were randomly selected for the study. All the instructions related to complete denture patient education and motivation were first congregated into nine different categories. $^{2,6-15}$ After careful evaluation these categories were designated into five main groups (Table 1) namely general instructions, treatment needs, specific, significant and miscellaneous instructions. The reason for categorizing instructions was to evaluate the degree of difficulty of remembering instructions while grouping was to simplify it for patients. All instructions were then translated into the local language following which the translated version was translated back into English for consistency. These instructions were then made available in the form of a written brochure and at the same time they were also labelled for ease of identification during recall by the patient. The patients were then treated for their conventional complete denture prosthesis by the post graduates of the department of Prosthodontics, under the supervision of staff having experience in the field of Prosthodontics for more than eight years. On the last day of denture fabrication all patients were given 
instructions verbally in a quiet and isolated room to avoid distraction, following which the patients were given a written brochure in the language they preferred. The verbal instruction session with patient on an average lasted for about forty five minutes. All patients were asked to report one day and one month after delivery of prosthesis respectively, so that short and long term response could be measured.

On the day of each recall, the patients were asked to recall the instructions that were verbally as well as given in written to them. At the first appointment, all patients were given a denture hygiene maintenance kit which included a denture box (NMS Nirmala Ltd), denture brush (Stim) and denture cleansing powder (ICPA Health Products Ltd). At second recall appointment patient's denture was examined for denture hygiene through a denture plaque index. ${ }^{16,17}$ Denture plaque index determined patient's motivation and compliance with denture hygiene instructions. Denture plaque levels were examined by using the Red 3 Erythrosine dye disclosing agents.

Data analysis included determination of mean and standard deviation along with standard difference for each category of instructions belonging to their relevant group at first and second appointments. Paired ' $\mathrm{t}$ ' test was applied for comparisons between the first and second appointment between male and female subjects. Statistical significance was determined at $p<.05$ level and was performed using SPSS version 20.

\section{Results}

The results were evaluated by first determining the mean with standard deviation for each category under each group at both first and second appointments (Table 2). After calculating the mean differences and the SD differences, paired ' $\mathrm{t}$ ' test was applied to know the significance of each mean value for each category at both appointments. The mean for total no of instructions recalled at first appointment was 10.9 whereas for second appointment it was 17.6. Instructions in group D (Significant instructions) were recalled most at both appointments. Application of paired ' $t$ ' test shows that significant values were obtained for all categories except category $6(\mathrm{p}=0.37), 8(0.58)$ and $9(0.32)$ which were grouped under specific, significant and miscellaneous instructions (Table 2). Gender differences were observed at first follow up where male subjects showed better recall than female subjects except for group D (significant) instructions (11\% as compared to $13 \%$ of female subjects) although the total percentage of recalled instructions were poor for both sexes (Graph 1). At the second appointment, female subjects showed better recall of instructions than male subjects for group $\mathrm{A}$ and B. Instructions in group $\mathrm{C}$ and $\mathrm{E}$ show poor recall by both sexes. To evaluate the effectiveness of the hygiene instructions that were given, the denture plaque index scores were recorded and according to the score obtained, the subjects were distributed under three categories namely good, average and poor hygiene (Graph 2). Whereas only $27 \%$ of total subjects scored good, a large portion of both male (33\%) and female (36\%) had denture hygiene index that was termed as poor.

\footnotetext{
Table 1: List of Instructions

Category 1, 2 and 3 (Group A) - General instructions (n=18)

Category 4 and 5 (Group B) - Instructions associated with Treatment needs $(n=15)$

Category 6 (Group C) - Specific instructions $(n=5)$

Category 7 and 8 (Group D) - Significant instructions $(n=26)$

Category 9 (Group E) - Miscellaneous (n=3)

Group A (General instructions)

- Natural teeth have roots and each tooth has its own movement, whereas in complete denture all the teeth rest on one denture base which acts as a single unit.

- Complete dentures cannot replace natural teeth in all respects they are just a solution for patients with no teeth.

- Although complete denture is a solution for loss of all the teeth, but it has its own set of limitations, e.g. taste of acrylic, wear, shrinkage water sorption.

- Problems with complete dentures can be overcome only with patience, determination and skill. The success of the dentures rests with the patient's ability to understand the basic limitations associated with complete dentures

- Minute changes or variation in the movement of a body that is present in between the teeth cannot be detected by the complete denture like rolling of a peanut within two teeth

- Ability to detect minute variation in sizes e.g. peanut vs pea, is absent in the denture.

- Ability to detect differences in location is absent in the denture. e.g. where the food is present between the teeth
} 
- The difference in texture, i.e. difference between the fibre of flesh and fibre of sugar cane cannot be detected by dentures

- Average biting force of natural teeth is $20 \mathrm{~kg}$ whereas in complete dentures it is $6-8 \mathrm{~kg}$ i.e. $1 / 5^{\text {th }}$ of the natural teeth

- Teeth are 32 individual units, whereas dentures are only 2 units and within these 2 units if force is applied on one side, it will affect the other side of the denture

- When the dentures are inserted for the first time, you may experience a temporary feeling of strained face

- At first, facial expression may seem to be altered because of the sudden change. It will take some time for the muscles and lips to relax and assume their natural position around denture

- You may become aware of bulk and you will have a feeling of unusual fullness. This may develop in nausea or gagging sensations especially if you are nervous

- Lip over the anterior teeth may feel protuberant. This feeling will disappear as you keep on using the dentures

- Do not insist that the upper denture is too long. Anatomic landmarks determine the exact length of the denture and the seal or suction will be destroyed if the denture is shortened. If this problem occurs, consult your dentist, but remember this is a problem that you must largely overcome through perseverance

- Salivation is a normal body process. It aids in lubrication, digestion and swallowing of food. Initially for 1-2 days salivary flow will increase, but it will reduce slowly over a period of time.

- The saliva should not be allowed to collect with in the mouth and should be swallowed repeatedly to get rid of it.

- Irrational rinsing or spitting should be avoided.

Group B (Treatment needs)

- Initially, as you feel crowding of the tongue or fullness of the mouth, your speech will be altered

- During the rapid conversation, fluency of the speech may be affected and uncoordinated.

- A good way to learn is by reading aloud before a mirror.

- Any alteration in your speech will be more obvious during speaking of sounds. e.g./s/,/z/,/f/

- Do not pronounce the same word or sounds repeatedly

- Eating with denture is a skill which is slowly developing and need to be learned.

- Initially limit to soft foods or crispy foods that are easily broken.

- Sticky food should be avoided with dentures.

- Take small bites of food, chew each bite thoroughly, and avoid swallowing with too much liquid.

- Divide the normal food into small halves and place one half on each side between the posterior teeth

- Chewing stroke should be up and down and not side to side.

- You must eat food that requires little chewing i.e those ready for swallowing with a simple push of the tongue. e.g. rice

- During the learning period, avoid eating with friends or family.

- Place food towards corner of the mouth and not entirely, i.e front teeth.

- Initially there may be alteration in the taste of the food.

Group C (Specific instructions)

- Do not compare your new denture improvement with others denture experience as physical, mental and oral condition of each patient is individual in nature.

- Avoid exhibiting your dentures to curious friends till you are confident and comfortable at exhibiting them

- Problems are time dependent which means they will pass with time and learning.

- Do not overexpose yourself for the reason of getting a new denture

- Ability to adapt to new denture changes with age.

Group D (Significant instructions)

- Dentures should not be worn continuously initially.

- Gradually increase the number of wearing hours/day, i.e maximum of 8 hours.

- Dentures are to be removed before sleep so as to provide adequate tissue rest.

- Oral tissues were never intended to be covered, or squeezed by a hard denture base.

- Residual ridges should be rubbed with fingers or a wash cloth. It will remove the accumulated debris

- Clean the tongue with brush as it will reduce the inflammation. Brushing the tongue will decrease the loss of taste perception.

- It is essential for you to have a regular and balanced intake of essential nutrients for the maintenance of oral epithelium.

- Do not drink excessive alcohol as it will decrease tissue tolerance, alter denture surface and impair neuromuscular control and coordination.

- Brush the dentures with soft brush under running water.

- Do not use stiff brushes for cleaning the dentures.

- Clean the dentures and rinse the mouth after each meal.

- Once a day, dentures should be soaked in a cleanser for $30 \mathrm{~min}$.

- The denture should be brushed over a basin partially filled with water or covered with wet cloth.

- Sit down and wash it to prevent the breakage.

- Do not use toothpastes because it contains abrasives which may abrade the dentures.

- If any irritation of tissue is experienced, patients are advised to remove their dentures and rest the mouth for a while and report to the doctor.

- When dentures are left out of mouth, they should be placed in a container with water to prevent warpage

- Water of the container should be changed daily.

- The container should be covered with a lid.

- To remove calculus, overnight soaking with 4 ounces (114cc) white vinegar (occasional).

- Keep the denture away from children and/or animals as they may damage it

- The tongue has to be kept in a position that is important for the stability of the denture as stability of mandibular denture depends upon the proper tongue position.

- The maxilla has more surface area than mandible, but occlusal loading is equal, moreover the musculature around the lower denture exerts additional force to the mandible denture.

- Practice opening and closing while the tongue assumes an ideal position

- The contours of the denture flanges depend upon the contours of the tongue and the surrounding musculature.

- Improper tongue position, after demonstrating, is a patient's problem and no denture adjustment is needed.

Group E (Miscellaneous)

- Do not use any household remedy or do it yourself repairs of any sort like the use of gums.

- Because of the inherent problems of acrylic like water sorption, shrinkage and continual change in tissues periodic follow up is a must.

- There are responsibilities which we both have to fulfill 
Table 2: Number of instructions recalled by both subjects under various categories in all groups at both appointments

\begin{tabular}{|c|c|c|c|c|c|c|c|c|c|c|c|}
\hline & & \multicolumn{9}{|c|}{ Category number/ instructions given in each category } & \\
\hline & & \multicolumn{3}{|c|}{$\begin{array}{c}\quad(\mathbf{G p}-\mathbf{A}) \\
\text { General Instructions }\end{array}$} & \multicolumn{2}{|c|}{$\begin{array}{l}(\mathbf{G p}-\mathbf{B}) \\
\text { Instructions related to } \\
\text { treatment needs }\end{array}$} & \multirow{2}{*}{$\begin{array}{c}\mathbf{G p}-\mathbf{C}) \\
\text { Specific } \\
\text { Instructions } \\
\text { C-6 (5) }\end{array}$} & \multicolumn{2}{|c|}{$\begin{array}{l}\text { (Gp-D) } \\
\text { Significant } \\
\text { Instructions }\end{array}$} & \multirow{2}{*}{$\begin{array}{c}\mathbf{G p}-\mathbf{E}) \\
\text { Misc. } \\
\text { C-9 (3) }\end{array}$} & \\
\hline $\begin{array}{l}\text { Category/numb } \\
\text { er of } \\
\text { instructions }\end{array}$ & Summary & C-1 (10) & $\mathrm{C}-2(5)$ & C-3 (3) & C-4 (5) & C-5 (10) & & C-7 (20) & $\mathrm{C}-8(6)$ & & $\begin{array}{c}\text { TOTAL } \\
67\end{array}$ \\
\hline \multirow[t]{2}{*}{ Appointment I } & Mean & 0.4333 & 1 & 1.2667 & 0.6 & 2.5333 & 0.1667 & 4.6333 & 0.2 & 0.0667 & 10.9 \\
\hline & Std Dev. & 0.9714 & 0.7428 & 0.8277 & 0.8137 & 1.1958 & 0.379 & 1.8843 & 0.8052 & 0.2537 & 5.5977 \\
\hline \multirow[t]{6}{*}{ Appointment II } & Mean & 0.8667 & 1.9667 & 1.9667 & 1.2 & 3.7 & 0.2667 & 7.4667 & 0.3 & 0.0333 & 17.6 \\
\hline & Std Dev. & 0.8604 & 0.6687 & 0.4901 & 1.0954 & 1.2077 & 0.5833 & 1.9605 & 0.4661 & 0.1826 & 4.4225 \\
\hline & Mean diff & -0.4333 & -0.9667 & -0.7000 & -0.6000 & -1.1667 & -0.1000 & -2.8333 & -0.1000 & 0.0333 & -6.7000 \\
\hline & SD diff & 1.1043 & 0.7649 & 0.9523 & 1.1326 & 1.5775 & 0.6074 & 2.6272 & 0.9948 & 0.1826 & 6.8740 \\
\hline & $\begin{array}{l}\text { Paired t- } \\
\text { value }\end{array}$ & -2.1492 & -6.9221 & -4.0261 & -2.9016 & -4.0508 & -0.9017 & -5.9069 & -0.5506 & 1.0000 & -5.3386 \\
\hline & p-value & $0.0401 *$ & $0.0000^{*}$ & $0.0004^{*}$ & $0.0070^{*}$ & $0.0003^{*}$ & 0.3746 & $0.0000^{*}$ & 0.5861 & 0.3256 & $0.0000^{*}$ \\
\hline \multicolumn{12}{|c|}{$* \mathrm{p}<0.05$ shows significant result } \\
\hline \multicolumn{12}{|c|}{$\begin{array}{l}\text { Table 2: Paired ' } t \text { ' test applied for comparison between the } 1^{\text {st }} \text { and the } 2^{\text {nd }} \text { appointment recall of instructions shows significant difference at the } 0.05 \text { level of } \\
\text { significance with respect to the category } 1 \text { (nature of complete dentures), } 2 \text { (first oral feeling), } 3 \text { (increased salivation), } 4 \text { (speech), } 5 \text { (eating with dentures), and } 7 \\
\text { (maintenance of denture hygiene). No significant difference was found for the category } 6 \text { (individuality of the patient), } 8 \text { (tongue position) and } 9 \text { (miscellaneous). }\end{array}$} \\
\hline
\end{tabular}

\section{Comparative gender differences for recalling instructions at first and second appointments}

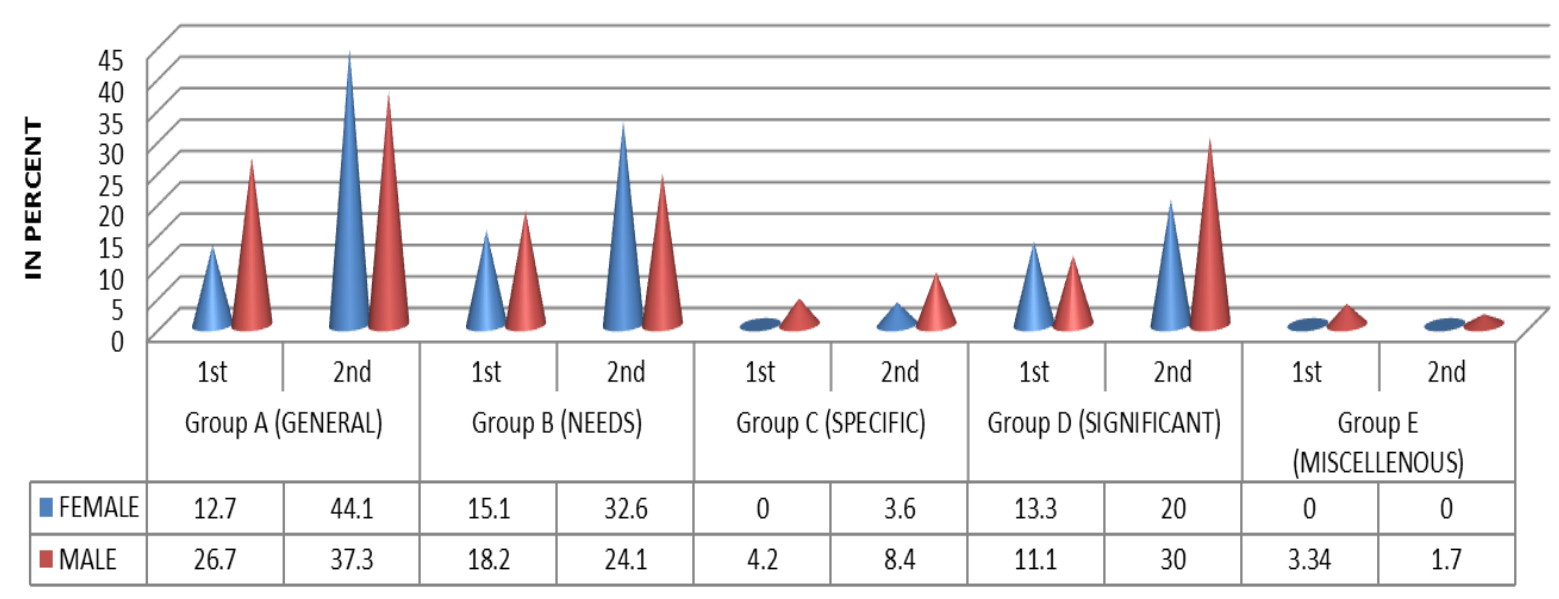

Graph 1: Instructions (frequency) recalled within five different groups at first and second appointment between male and female subjects

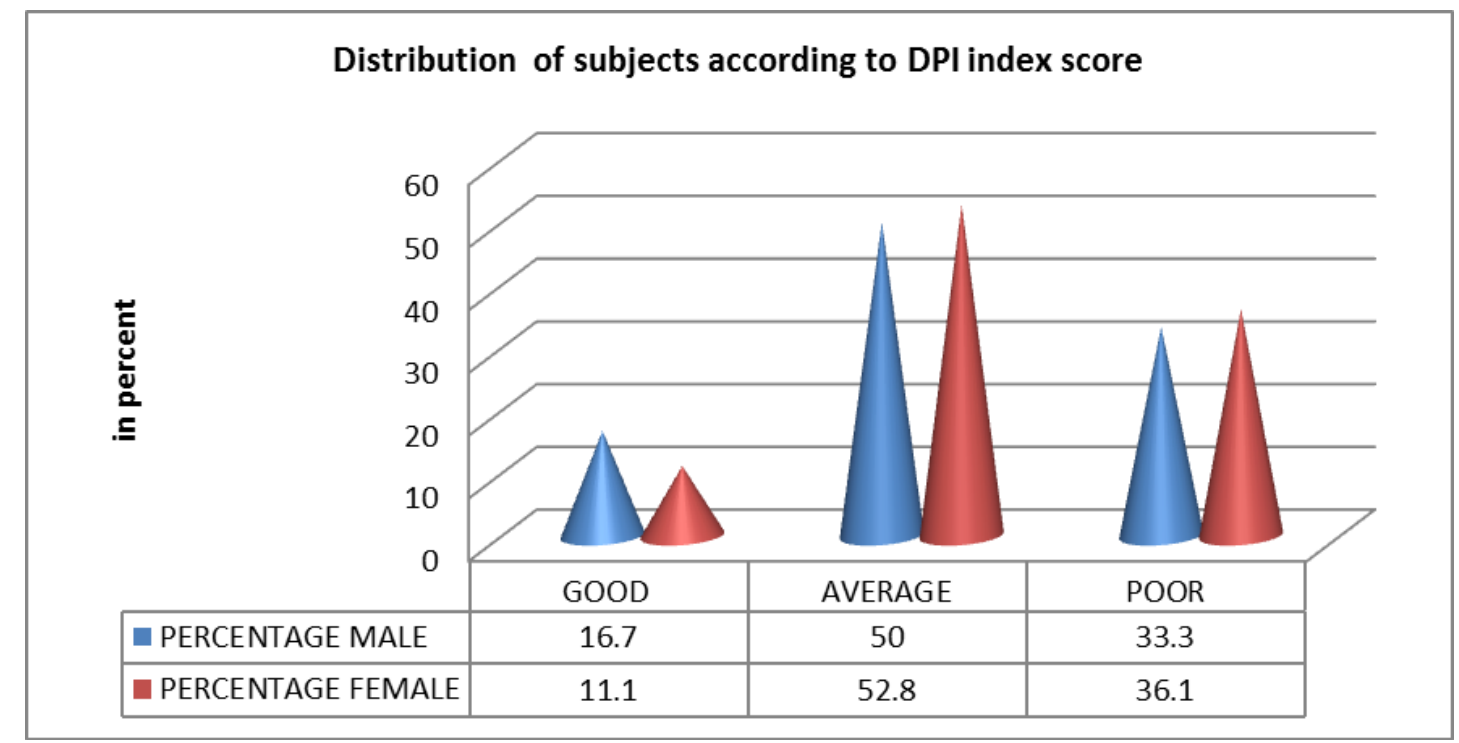

Graph 2: Overall denture hygiene evaluation of all subjects at second recall visit 


\section{Discussion}

Effective communication with all patients is the fundamental requirement for proper care. For dentally handicapped geriatric patients, it is even more so because their motivation is so variable, and it is therefore more difficult to achieve the accuracy. ${ }^{18}$ Complete denture prosthesis in any of its forms is the most common prescription offered to the completely edentulous patient in occlusal rehabilitation. However, ensuring prosthetic success requires an understanding of both the patient and the dentist related factors. ${ }^{19}$ Studies in dentistry addressing the connection among dentist/patient characteristics, communication, and outcome are scarce. There is no previous study of this issue in prosthetic dentistry. ${ }^{20}$ Adaptation of the patient to the prosthesis is an important criterion for success. No matter how simple or complicated prosthesis is, it requires patients to understand its limitations and restrictions. This is achieved through proper instructions, given to the patient regarding prosthesis adaptation and maintenance. On the other hand, the patient's ability to recollect medical and dental instructions are often poor and inaccurate as the tendency is to focus on diagnosis-related information. Patients can be helped to remember medical information by the use of explicit categorization techniques. ${ }^{21}$ Ley asserted that notable information should be presented early on conversation and should be organized into specific categories. Organization is especially important as recall is negatively affected as a person ages. ${ }^{22}$ Also greater the amount of information presented, the lower the proportion correctly recalled. ${ }^{21,22}$ The present study was inspired after a survey revealed that the number of instructions given to complete denture patients on average by dental students was very less and the reason for that was the degree of difficulty of those instructions. In order to ease memorizing of instructions, nine categories were made which contained about sixty seven instructions. Amongst the various modes of information, verbal method along with written information have shown better treatment adherence.
The initial success of any prosthesis is determined by the duration of adaptation period. The time ranges from 3 to six months, but the important phase of this adaptation is patients following the instructions regarding maintenance and care for the prosthesis. First week after denture delivery is especially critical as it is during this phase the daily habits with the new prosthesis would be established. Therefore, it is important to evaluate the patient's ability to recall the given instructions within the first seven days. Expect category 8 (Gp-D), all other instructions in other categories need to be practiced soon after denture delivery. The most common reasons that the patients were seeking complete denture treatment were inability to masticate and impaired esthetic reasons which is in accordance to a study by Roessler. ${ }^{19}$ Therefore, instructions related to eating with dentures tend to be listened carefully by the patients. The results can be related to the study done by Witt and Bartsch (1993) who found that the information regarding treatment needs, possible side effects and risks had a better recall. ${ }^{23}$ As the recall of information in these categories was found to be sufficiently high it is possible, however, that subjects were particularly attentive by virtue of the fact that they were taking part in a study. ${ }^{24}$ Group $\mathrm{C}$ and group $\mathrm{E}$ instructions could not be recalled by the patient. Queries regarding these groups revealed that most of the patients did not have that social interaction with other people because they have adapted to a different life style. Most of the male patients revealed they prefer to stay at home, watch television and most of the social conversations were being carried on through cellular phones. Therefore, they would tend to interact less even when they would receive new dentures. Among female patients there was one subject who did not display the dentures to people who had visited her house because she was feeling shy and was apprehensive of the fact that they might notice her dentures. Decline in recall of instructions was noticed in 9 patients between first and second appointment in group B and D. One of the most common complaint of complete denture patients concern the "loose" mandibular dentures. ${ }^{12}$ Many 
of these problems stem from a patient's lack of understanding and relating the special problems associated with the mandibular dentures. ${ }^{12}$ The results of the study also show that instructions regarding denture hygiene maintenance were aptly followed by the majority of the patients, indicating that they are easy to learn and practice and the results are immediately evident to the patient.

\section{Conclusion}

Within the limitations of this in vivo study it can be concluded that the degree of difficulty of remembering instructions regarding any prosthetic treatment should be established first especially the categories to which they would belong. Perseverance on the part of the prosthodontist is required to instill education of the patient regarding the prosthesis he would have to adapt to and it requires more than one appointment. Future research should establish additional modes of delivering all instructions. More research is needed to find out the reasons as to why female patients showed more improvement in recall of instructions than their male counterpart and such methods could be used in clinical settings to improve patient compliance.

\section{Practical Implication}

Most dissatisfied patients who term their treatment as unsuccessful can be avoided if the things they are expected to do is communicated to them properly. With advances in multimedia and communicating tools we can minimize these justified errors that still lie in our practice.

\section{Acknowledgements}

The authors would like to acknowledge all the subjects that participated in the study and all the postgraduate students of the department of Prosthodontics who vehemently followed the treatment protocol for all the patients.

\section{References}

1. Zlataric DK, Celebic A. Treatment outcomes with removable partial dentures: a comparison between patient and prosthodontist assessments. Int J Prosthodont 2001; 14:423-6.

2. Jacob RF, Zarb GA, Bolender CL. Waxing and processing the dentures, their insertion and follow up. In: Zarb-Bolender. 12th ed. Prosthodontic treatment of edentulous patients; 2003: 414-9.

3. Sondell K, Palmqvist S, Soderfeldt B. The dentist's communicative role in prosthodontic treatment.Int $\mathrm{J}$ Prosthodont 2004;17:666-71

4. Guckes AD, Smith DE, Swoope CC. Counselling and related factors influencing satisfaction with dentures. J Prosthet Dent.1978; 39 (3): 259-67.

5. World Medical Association Declaration of Helsinki: ethical principles for medical research involving human subjects. JAMA. 2013 Nov 27;310 (20): 2191-4

6. Schalbel RW. Patient education with prosthetic acrylic resin models. J Prosthet Dent 1967; 17 (2): 106-8.

7. Maison WG. Instructions to the denture patient. J Prosthet Dent.1959; 9 (5): 825-31.

8. Swenson MG. Neglected factors in denture service. J Prosthet Dent. 1951; 1:71-7.

9. Boos RH. Preparation and conditioning of patients for prosthetic treatment. J Prosthet Dent. 1959; 9(1):4-10.

10. Vere AM. Denture education for edentulous patients. J Prosthet Dent. 1966; 16(6):10138.

11. Jeganathan S. Common faults in complete dentures: a review. Quintessence Int 1993; 24:483-7.

12. Ortman LF. Patient education and complete denture maintenance. In: Sheldon Winkler. 2nd ed. Essentials of complete denture Prosthodontics; 1996: 331-40.

13. Educating the patient. In: Rahn AR, Heartwell CM. 5th ed. Textbook of complete dentures; 2002:121-30. 
14. Kingery. The post insertion phase of denture treatment. D Clin North America 1960: 34358.

15. Budtz-Jorgensen. Materials and methods for cleaning dentures. J Prosthet Dent 1979; 42 (6): 619:23.

16. McCabe JF. A method for scoring plaque. Eur J Prosthodont Restor Dent.1996; 4(2):59-64.

17. Jeganathan S, Chan YC. A clinically viable index for quantifying denture plaque. Quintessence Int 1996; 27: 569-73.

18. Anastassiadou V, Katsouli S, Heath MR, Pissiotis A and Kapari D. Validation of communication between elderly denture wearers and dentists: a questionnaire on satisfaction with complete dentures using semi-structured interviews. Gerodontology 2004; 21; 195-200

19. Roessler DM. Complete denture success for patients and dentists. International Dental Journal 2003; 53: 340-5.

20. Sondell K. Dentist patient communication and patient satisfaction in prosthetic dentistry. Int J Prosthodont 2002; 15: 28-37.

21. Kessels PC. Patients' memory for medical information. J R Soc Med 2003; 96: 219222.

22. Zirwas MJ. Patient education strategies in Dermatology- part 1: benefits and challenges. J Clin Aesthetic Dermatol 2009; 2 (12): 24-7.

23. Witt E. and Bartsch A. (1993). The effect of information and communication in the orthodontic consultation. Part 1: the imparting of information. Fortschritte der Kieferorthopädie, 54, 187-195

24. Thompson AM. A comparison of information retention at an initial orthodontic consultation. European Journal of Orthodontics.2001; 23:169-78. 